\section{Highly reproducible tunnel currents in MBE-grown semiconductor multilayers}

\section{Shao, P. Dasmahapatra, J. Sexton, M. Missous and} M.J. Kelly

Tunnel currents through semiconductor tunnel barriers have proved very difficult to control to the extent that device-to-device variability and wafer-to-wafer irreproducibility have prevented electronic devices based on tunnelling from ever going into production. With reference to a single tunnel barrier of AlAs in a GaAs multilayer structure with an asymmetric doping profile, it is shown that, with careful attention to detail, diodes from equivalent sites on three separate wafers can be produced whose average current in forward bias is within $1 \%$ while the total in-wafer standard deviation of current at the same fixed bias $(0.5 \mathrm{~V})$ is $6 \%$, dominated by a systematic crosswafer variation that is described. This level of reproducibility now enables these devices to be used in pick-and-place systems for the manufacture of low-cost hybrid integrated microwave circuits.

Introduction: For 30 years now, a whole range of semiconductor multilayer devices that incorporate thin tunnel barrier layers have demonstrated on a one-off basis a superior performance in transistors, lasers, microwave and infrared sources and detectors, and they also allow multi-level logic to be incorporated to simplify the transistor count of circuits such as adders [1]. None of these devices has entered mass production, and the one common reason has been the inability to achieve a sufficient level of reproducibility and uniformity of the tunnelling current from device to device within a wafer and from wafer to wafer to allow the techniques of low-cost manufacture to be deployed. The reason for the extreme sensitivity of currents through semiconductor tunnel barriers was most thoroughly shown six years ago [2], and by exploiting the conditions laid out in that paper, we have been able to eliminate this sensitivity in a special set of growth conditions, by growing the tunnel barrier to be precisely an integer number of monolayers thick.

Fabrication of ASPAT diode: A single AlAs tunnel barrier, placed towards one end of the i-region of an n-i-n GaAs structure acts as a rectifying diode, the asymmetric spacer layer tunnel (ASPAT) diode [3], with some very attractive characteristics as a microwave detector. It has the same basic transfer efficiency (the terminal voltage achieved for a fixed input power) as other diodes, but it is a low noise device, with very low sensitivity to ambient temperature and it operates as a zero-bias detector. Textbook examples of electron tunnelling currents through thin layers show an exponential sensitivity to barrier layer thickness [4], and for a typical semiconductor, a one layer variation in thickness means a $300 \%$ variation in tunnelling current. Over the 20 years since the original discovery, the attempts to establish a manufacturable process have foundered on the intrinsic device-to-device fluctuations, as we were seeing the effects of alloy monolayers at the interfaces with spreads as much as $100 \%$ in the performance of devices from wafer to wafer and 30-50\% variations in-wafer $[5,6]$.

A multiple wafer run (wafers VMBE2067 A, B and C) used for fabrication of the ASPAT were grown following the ex-situ re-calibration technique reported earlier [6], and using a V90H molecular beam epitaxy (MBE) machine (manufactured by RIBER). The three wafers have an epi-layer profile as follows (with silicon as the dopant, and $\mathrm{NID}=$ not intentionally doped)

\begin{tabular}{|c|c|c|c|}
\hline Layer & Material & Thickness $(\mathrm{nm})$ & Doping $\left(\mathrm{cm}^{-3}\right)$ \\
\hline 7 & GaAs & 300 & $4 \times 10^{18}$ \\
\hline 6 & GaAs & 40 & $1 \times 10^{17}$ \\
\hline 5 & GaAs & 5 & NID \\
\hline 4 & AlAs & 2.84 & NID \\
\hline 3 & GaAs & 200 & NID \\
\hline 2 & GaAs & 40 & $1 \times 10^{17}$ \\
\hline 1 & GaAs & 350 & $4 \times 10^{18}$ \\
\hline 0 & GaAs & substrate & $\mathrm{n}+$ \\
\hline
\end{tabular}

The key point for these experiments was the necessity to achieve precisely 10 integer monolayer AlAs barrier thicknesses. This was done through both the pre-calibration procedure immediately before the growth of the ASPAT wafer but more importantly through use of a very low growth rate for AlAs to ensure tight control over the thickness. The mechanical Al shutter control (within $0.1 \mathrm{~s}$ between opening and shutting) could in theory contribute $2 \%$ of one monolayer fluctuation. The substrate rotation, which is kept at $\sim 30 \mathrm{rpm}$, ensured that the deposition time for a monolayer $(\sim 6 \mathrm{~s})$ eliminates any systematic variation in barrier thickness across the wafers, other than a linear and radial effect, actually seen below because of the off-axis geometry of the Knudsen cells within the MBE chamber.

Devices were fabricated on the three 2 -inch $\mathrm{n}^{+}$-doped substrates using a full wet-etch process. Epitaxial layers were first etched using a nonselective ortho-phosphoric based etchant $\mathrm{H}_{3} \mathrm{PO}_{4}: \mathrm{H}_{2} \mathrm{O}_{2}: \mathrm{H}_{2} \mathrm{O}$ (3:1:50) to isolate the ASPAT. Front and back alloyed ohmic contacts constituted of $5 \mathrm{~nm} \mathrm{AuGe}, 12.5 \mathrm{~nm} \mathrm{Ni}$ and $200 \mathrm{~nm}$ Au were thermally evaporated and annealed at $415^{\circ} \mathrm{C}$ for $1 \mathrm{~min}$. This provided a low $(<0.1 \Omega . \mathrm{mm})$ contact resistance as determined from the transmission line method (TLM) characterisation study and thus its contribution to the overall device resistance is negligible. The full-wafer layout consisted of a total of 16 fields with six intentionally left blank for future testing studies. Within the 10 used fields, a total of 456 devices (per field) with mesa dimensions of $100 \times 100 \mu^{2}$ down to $5 \times 5 \mu \mathrm{m}^{2}$ were available to be manually measured for this study.

Results: The main results we wish to report here are on the uniformity and reproducibility of the devices. We used a Cascade probe station to measure the average and standard deviation of the forward bias current from 30 devices from the same positions in the same block of devices within the same field on each of different wafers, each mesa of $100 \mu \mathrm{m}$ side. We obtained the same average current to within $1 \%$ at $0.5 \mathrm{~V}$ forward bias from all three wafers (see Fig. 1), and the standard deviation of $3 \%$ within the local block. Note that these variations also include any variation coming from the probe technique and the ohmic contact resistances. This percentage variability is then halved to obtain the uniformity of the curvature of the diode $I-V$ characteristic near the origin, which is a direct measure of the key figure of merit of the diode as a microwave detector, namely its transfer efficiency, i.e. the terminal voltage induced by a given level of input microwave power. The forward bias experimental data was also within 1-2\% agreement with the predictions of the Silvaco (C) software used in the device design.

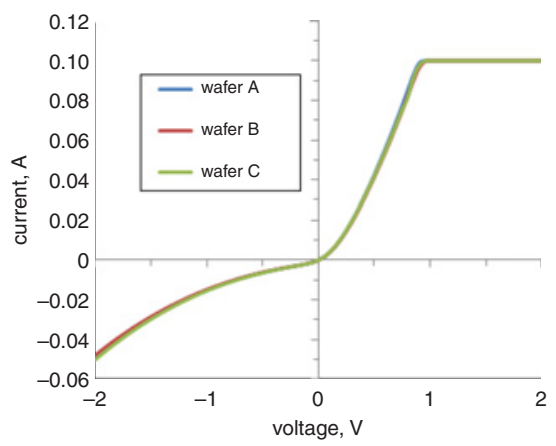

Fig. 1 Average I-V characteristics of 30 diodes across same one block left on bottom row in Fig. 2 below) of diodes in each of three wafers

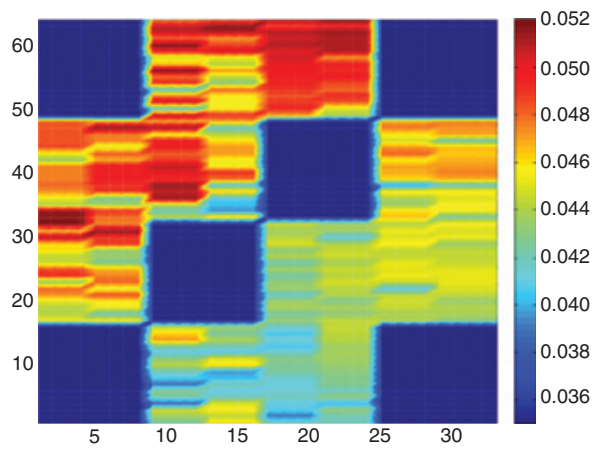

Fig. 2 Map for wafer $B$ of current at $0.5 \mathrm{~V}$ forward bias on 400 devices across wafer, showing gradual increase in current (and thinning of barrier by $\sim 0.06$ monolaver) across wafer

The flat of wafer at bottom of map, and all three wafers, disposed symmetrically on holder, show same qualitative and quantitative behaviour. Note that there are 10 fields of identical devices, with six fields blank for other purposes 
Fig. 2 shows a colour contour of the currents at $0.5 \mathrm{~V}$ forward bias for wafer B but all show a qualitatively and quantitatively similar pattern. The percentage standard deviations of the forward bias current of typically 60 devices in each of the ten fields, of each of the three wafers, were between 3 and 7\%. These incorporate processing variations, probing variations and, as can be seen from Fig. 2, what is a dominating contribution from the (linear) gradation of current across the wafer from bottom to top. This last effect is of great interest, as it reflects the ultimate limitations of the geometry of the growth chamber.

In Fig. 2, the flat of the wafer is at the bottom, and is nearest the centre of the platen that holds the wafers during growth, typically $3 \mathrm{~cm}$ from the axis about which the platen rotates during growth. The lower current indicates a thicker barrier. The top of the Figure comes from the opposite side of the wafer which rotates at $8 \mathrm{~cm}$ from the platen axis, and the higher current indicates a thinner barrier. We have been able to model this variation. There is a $25 \%$ rise in current across the wafer, and this represents a $<0.1$ monolayer decrease in barrier thickness (simulations indicate a $300 \%$ fall in current for an extra monolayer of AlAs in the barrier): this is a $<1 \%$ variation across the wafer in the barrier thickness which is intended to be 10 monolayers thick. The aluminium beam intensity is rate-determining for the growth of AlAs barriers (as there is an over-pressure of arsenic). From the off-axis position of the Knudsen cell containing $\mathrm{Al}$, and its pointing towards a point on the axis at or below the centre of the platen, we can map out the $\cos (\theta)$ variation of the beam intensity at any instant at any place on the platen, and average this over one cycle to get the dependence of the average Al beam intensity. This can be done analytically and if the beam is focused $10 \mathrm{~cm}$ below the wafer centre, a $1.5 \%$ variation in thickness across the wafer is predicted given the geometry of the growth chamber in close agreement with what is observed. This can clearly be reduced in a larger growth chamber, and with a redesign of the wafer holder. By contrast the low level of side-to-side variation of the tunnelling current seen in Fig. 2 is consistent with wafer uniformity approaching 0.02 monolayers. This is a much greater precision than previously reported anywhere.

Conclusion: We have achieved levels of precision and reproducibility not achieved before in the attempts to manufacture tunnel devices.
With design and growth focusing on precisely integer monolayer growth in MBE machines, all devices listed in the Introduction Section are capable of manufacture.

(C) The Institution of Engineering and Technology 2012 10 April 2012 doi: 10.1049/el.2012.1214

One or more of the Figures in this Letter are available in colour online. C. Shao and M.J. Kelly (Centre for Advanced Photonics and Electronics, Department of Engineering, University of Cambridge, 9 JJ Thomson Avenue, Cambridge, CB3 OFA, United Kingdom)

E-mail:mjk1@cam.ac.uk

P. Dasmahapatra, J. Sexton and M. Missous (The School of Electrical and Electronic Engineering, The University of Manchester, Sackville Street, Manchester, M60 1QD, United Kingdom)

\section{References}

1 Eaves, L., and Kelly, M.J. (Eds): 'The current status of semiconductor tunnelling devices', Phil. Trans. Roy. Soc., 1996, 354, pp. 2289-2467

2 Kelly, M.J.: 'The unacceptable variability in tunnel currents for proposed electronic device applications', Semicond. Sci. Technol., 2006, 21, pp. L49-51

3 Syme, R.T., Kelly, M.J., Smith, R.E., Condie, A., and Dale, I.: 'A tunnel diode with asymmetric spacer-layers for use as a microwave detector', Electron. Lett., 1991, 27, pp. 2192-4 and Syme, R.T.: 'Microwave detection using GaAs/Alas tunnel structures,' GEC J. Res., 1993, 11, pp. $12-23$

4 Landau, L.D., and Lifshitz, E.M.: 'Quantum mechanics', Vol. 3, 3rd edn (revised) of 'A course in theoretical physics' (Pergamon, 1977)

5 Billen, K., Wilkinson, V.A., and Kelly, M.J.: 'Manufacturability of heterojunction tunnel diodes: further progress', Semicond. Sci. Technol., 1997, 12, pp. 894-898

6 Hayden, R.K., Gunnaes, A.E., Missous, M., Khan, R., Kelly, M.J., and Goringe, M.J.: 'Ex-situ re-calibration method for low-cost precision epitaxial growth of heterostructure devices', Semicond. Sci. Technol., 2002, 17, pp. 135-140 